\title{
Open Access and Article Processing Charges in Cardiology and Cardiac Surgery Journals: a Cross- Sectional Analysis
}

Dominique Vervoort ${ }^{1}$, MD, MPH, MBA; Jessica G.Y. Luc ${ }^{2}$, MD; Michel Pompeu B. O. Sá3 ${ }^{3}$ MD, MSc, PhD; Eric W. Etchill ${ }^{4}$, $\mathrm{MD}, \mathrm{MPH}$

\begin{abstract}
Introduction: Open access (OA) publishing often requires article processing charges (APCs). While OA provides opportunities for broader readership, authors able to afford APCs are more commonly associated with well-funded, high-income country institutions, skewing knowledge dissemination. Here, we evaluate publishing models, OA practices, and APCs in cardiology and cardiac surgery.

Methods: The InCites Journal Citation Reports 2019 directory by Clarivate Analytics was searched for "Cardiac and Cardiovascular Systems" journals. Sister journals of included journals were identified. All journals were categorized as predominantly cardiology or cardiac surgery. Publishing models, APCs, and APC waivers were defined for all journals.

Results: One hundred sixty-one journals were identified (139 cardiology, 22 cardiac surgery). APCs ranged from \$244 to $\$ 5,000$ (\$244-5,000 cardiology; $\$ 383-3,300$ cardiac surgery), with mean $\$ 2,911 \pm 891$ and median $\$ 3,000$ (interquartile range [IQR]: $\$ 2,500-3,425)$ across 139 journals with non-zero available APCs
\end{abstract}

(\$2,970 \pm 890 , median $\$ 3,000$, IQR: $\$ 2,573-3,450$, cardiology; $\$ 2,491 \pm 799$, median $\$ 2,740$, IQR: $\$ 2,300-3,000$, cardiac surgery). Average APCs were \$3,307 \pm 566 and median \$3,250 (IQR: \$3,000$3,500)$ for hybrid journals $(\$ 3,344 \pm 583$, median $\$ 3,260$, IQR: $\$ 3,000-3,690$, cardiology; $\$ 2,983 \pm 221$, median $\$ 2,975$, IQR: $\$ 2,780$ 3,149 , cardiac surgery) and $\$ 1,997 \pm 832$ and median $\$ 2,100$ (IQR: $\$ 1,404-2,538)$ for fully OA journals $(\$ 2,039 \pm 843$, median $\$ 2,100$, IQR: \$1,419-2,604, cardiology; \$1,788 \pm 805 , median \$2,000, IQR: $\$ 1,475-2,345$, cardiac surgery). Waivers were available for 51 (86.4\%) fully OA and 37 (37.4\%) hybrid journals. Seventeen journals were fully OA without APCs, one journal did not yet release APCs, and four journals were subscription-only.

Conclusion: $O A$ publishing is common in cardiology and cardiac surgery with substantial APCs. Waivers remain limited, posing barriers for unfunded and lesser-funded researchers.

Keywords: Open Access Publishing. Access to Information. Publishing. Cardiac Surgical Procedures. Cardiology. Periodicals as Topic.

\begin{tabular}{|c|c|c|c|}
\hline \multicolumn{4}{|c|}{ Abbreviations, acronyms \& symbols } \\
\hline APCs & $=$ Article processing charges & JAMA & $=$ Journal of the American Medical Association \\
\hline BMC & = BioMed Central & JCR & $=$ Journal Citation Reports \\
\hline EP Europace & $\begin{aligned}= & \text { The European Journal of Pacing, Arrhythmias and } \\
& \text { Cardiac Electrophysiology }\end{aligned}$ & $\begin{array}{l}\text { JTCVS } \\
\text { OA }\end{array}$ & $\begin{array}{l}=\text { Journal of Thoracic and Cardiovascular Surgery } \\
=\text { Open access }\end{array}$ \\
\hline ESC & $=$ European Society of Cardiology & REC & = Revista Española de Cardiología \\
\hline IQR & $=$ Interquartile range & USD & = U.S. Dollar \\
\hline JACC & $=$ Journal of the American College of Cardiology & & \\
\hline
\end{tabular}

'Department of Health Policy and Management, Johns Hopkins Bloomberg School of Public Health, Baltimore, Maryland, United States of America.

2Division of Cardiovascular Surgery, Department of Surgery, University of British Columbia, Vancouver, British Columbia, Canada.

${ }^{3}$ Division of Cardiovascular Surgery, Pronto-Socorro Cardiológico de Pernambuco (PROCAPE), Universidade de Pernambuco, Recife, Pernambuco, Brazil.

${ }^{4}$ Department of Surgery, Johns Hopkins University School of Medicine, Baltimore, Maryland, United States of America.
Correspondence Address:

Dominique Vervoort

(iD) https://orcid.org/0000-0002-3142-0388

Department of Health Policy and Management, Johns Hopkins Bloomberg School of Public Health

615 North Wolfe Street, Baltimore, Maryland, United States of America

Zip Code: 21205

E-mail: vervoortdominique@hotmail.com 


\section{INTRODUCTION}

Scientific publishing is a multibillion-dollar industry, costing steep license fees for institutions to provide access to journals, high individual costs to subscribe to journals or buy articles, and little to no remuneration for reviewers' time. Authors not associated with institutions covering full or partial access to major journals are forced to find alternate methods of accessing publications, such as pirating, especially if unable to afford fees to access articles ${ }^{[1]}$. Similarly, authors are frequently charged article processing charges (APCs) to publish "open access" (OA). This further impedes early-career researchers, researchers from lesser-funded institutions, and researchers from most low- and middle-income countries to publish articles ${ }^{[2]}$. In response to high publishing fees, predatory journals are increasingly pervading scientific practice ${ }^{[3]}$. Predatory journals promise quick and easy OA publishing for a fee, typically at only a fraction of non-predatory journals, resulting in millions of dollars generated by these journals, even during the coronavirus disease 2019 (or COVID-19) pandemic ${ }^{[4]}$.

Despite the growing OA discourse, little is known regarding the most common subscription models and APCs. This study evaluates publishing models, OA practices, and APC amounts in cardiology and cardiac surgery journals.

\section{METHODS}

The InCites Journal Citation Reports (JCR) directory by Clarivate Analytics was searched to identify journals categorized as "Cardiac and Cardiovascular Systems" for 2019. Sister journals of included journals, defined as journals published by the same publisher and associated with the included journal, were further included. Journals were manually distinguished as being predominantly related to cardiology or to cardiac surgery based on their titles and, where applicable, associated professional societies. Publishing models (OA only, subscription only, or hybrid) and APCs, if applicable, were defined for all journals. OA only was defined as journals making all articles publicly available to readers, subscription only as journals making articles only available to readers with a (personal or institutional) subscription, and hybrid as a combination thereof. APCs were presented as mean \pm standard deviation and median with interquartile ranges (IQR). For journals with APCs, the availability of partial or full waivers as presented on the journal's or publisher's website was recorded.

\section{RESULTS}

In 2019, 137 non-duplicate journals were identified in the JCR Cardiac and Cardiovascular Systems category. Twenty-four journals were identified as sister journals of JCR-indexed journals for a total of 161 journals. One hundred thirty-nine journals were categorized as predominantly cardiology, of which 88 were hybrid, 49 fully OA, and two subscription. Twenty-two journals were categorized as predominantly cardiac surgery, of which 10 were hybrid, 10 fully $\mathrm{OA}$, and two subscription.

APCs ranged from $\$ 244$ to $\$ 5,000$ (\$244-5,000 cardiology; $\$ 383-3,300$ cardiac surgery), with an average of $\$ 2,911 \pm 891$ and median of $\$ 3,000$ (IQR: $\$ 2,500-3,425)$ across 139 journals with non-zero and available APCs $(\$ 2,970 \pm 890$, median $\$ 3,000$, IQR: \$2,573-3,450, cardiology; $\$ 2,491 \pm 799$, median $\$ 2,740$, IQR: $\$ 2,300-3,000$, cardiac surgery). Average APCs were $\$ 3,307 \pm 566$ and median $\$ 3,250$ (IQR: $\$ 3,000-3,500$ ) for hybrid journals (\$3,344 \pm 583 , median \$3,260, IQR: $\$ 3,000-3,690$, cardiology; $\$ 2,983 \pm 221$, median $\$ 2,975$, IQR: $\$ 2,780-3,149$, cardiac surgery) and $\$ 1,997 \pm 832$ and median $\$ 2,100$ (IQR: $\$ 1,404-2,538)$ for fully OA journals (\$2,039 \pm 843 , median $\$ 2,100$, IQR: $\$ 1,419-2,604$, cardiology; \$1,788 \pm 805 , median \$2,000, IQR: \$1,475-2,345, cardiac surgery). Waivers were available for 51 (86.4\%) fully OA journals, of which 16 (14 cardiology; two cardiac surgery) automatically applied waivers for authors from eligible low- and middle-income countries. Waivers were available for 37 (37.4\%) hybrid journals, none of which were automatically applied. Seventeen journals (14 cardiology; three cardiac surgery) were fully OA and did not charge APCs. Supplemental Tables 1 and 2 present findings for cardiology and cardiac surgery journals. All values are presented in U.S. Dollars.

\section{DISCUSSION}

OA publishing is widely available as hybrid or fully OA journals in cardiology and cardiac surgery, yet APCs vary from $\$ 244$ to $\$ 5,000$ per article. APC waivers were available for authors from eligible low- and middle-income countries, either automatically applied or on a case-by-case basis. Few journals considered financial need among authors from high-income countries, presumably with the assumption that authors would be able to find the means to cover the costs. Our findings suggest that, while the academic shift to $\mathrm{OA}$ is clearly visible ${ }^{[5]}$, enormous financial barriers on non-funded authors remain in the pursuit of $\mathrm{OA}$ publishing regardless of home country or institution. Although research on OA and APCs remains limited, lower average APCs were found in ophthalmology, multiple sclerosis research, and global health ${ }^{[6-8]}$.

While waivers were often considered by publishers, these were typically only considered when publishing in a fully OA journal. Most journals publishing as hybrid models did not have waiver availability, requiring authors to opt for subscription publication. Hybrid journals were on average $\$ 1,300$ more expensive than fully OA journals, which is confirmed by previous findings from research spending by North American institutions ${ }^{[9]}$. As these journals make up a majority of journals, especially those with higher impact factor, few authors are able to seek OA publishing of their work without having research funding or going through tedious processes of obtaining one-off external funding. Furthermore, while a majority of publishers clearly listed APCs, some publishers did not make APCs publicly available, which may deter and even deceive authors. Moreover, amongst the available waivers and discount criteria, researchers from uppermiddle-income countries find themselves in an "upper-middleincome trap", as they are commonly not eligible for such discounts or waivers despite lower income and standards of living compared to high-income countries. For example, authors from Brazil would not be eligible for discounts or waivers amongst the majority of main publishers (and thus journals); yet, there is vast variation in institutional and personal funding availability, with most researchers being unable to bear APCs. Lastly, APC waivers were typically 
Supplemental Table 1. Publishing models and article processing charges for cardiology journals indexed in InCites Journal Citation Reports.

\begin{tabular}{|c|c|c|c|c|}
\hline Journal Name & $\begin{array}{l}\text { Impact } \\
\text { Factor }\end{array}$ & $\begin{array}{l}\text { Publishing } \\
\text { Model }\end{array}$ & $\begin{array}{l}\text { Article Processing } \\
\text { Charges (USD) }\end{array}$ & Waivers Available \\
\hline Acta Cardiologica & 1.208 & Hybrid & 2,995 & No \\
\hline American Heart Journal & 4.153 & Hybrid & 3,250 & Case by case (Research4Life) \\
\hline American Journal of Cardiology & 2.570 & Hybrid & 2,960 & Case by case (Research4Life) \\
\hline American Journal of Cardiovascular Drugs & 2.674 & Hybrid & 3,860 & No \\
\hline $\begin{array}{l}\text { American Journal of Physiology: Heart and } \\
\text { Circulatory Physiology }\end{array}$ & 3.864 & Hybrid & 3,000 & No \\
\hline Anatolian Journal of Cardiology & 1.223 & Open Access & Free & - \\
\hline Annals of Noninvasive Electrocardiology & 1.131 & Open Access & 2,250 & Yes, upon request \\
\hline Annals of Thoracic Medicine & 1.456 & Open Access & Free & - \\
\hline Archives of Cardiovascular Diseases & 2.434 & Hybrid & 3,620 & Case by case (Research4Life) \\
\hline Arquivos de Brasileiros de Cardiologia & 1.450 & Open Access & Free & - \\
\hline Atherosclerosis & 3.919 & Hybrid & 3,200 & Case by case (Research4Life) \\
\hline Basic Research in Cardiology & 11.981 & Hybrid & 4,390 & No \\
\hline BMC Cardiovascular Disorders & 2.078 & Open Access & 2,380 & Case by case \\
\hline Canadian Journal of Cardiology & 5.234 & Hybrid & 3,200 & Case by case (Research4Life) \\
\hline Canadian Journal of Cardiology Open & - & Open Access & 2,100 & Automatic (Research4Life) \\
\hline Cardiology & 1.791 & Hybrid & 3,530 & No \\
\hline Cardiology Clinics & 1.811 & Hybrid & 3,150 & Case by case (Research4Life) \\
\hline Cardiology in Review & 1.816 & Hybrid & 2,400 & No \\
\hline Cardiology in the Young & 1.000 & Hybrid & 3,160 & No \\
\hline Cardiology Journal & 1.669 & Open Access & 900 & Case by case \\
\hline Cardiology Research and Practice & 1.292 & Open Access & 2,100 & Automatic \\
\hline Cardiorenal Medicine & 1.754 & Open Access & 2,580 & Case by case \\
\hline $\begin{array}{l}\text { Cardiovascular and Interventional } \\
\text { Radiology }\end{array}$ & 2.034 & Hybrid & 3,860 & No \\
\hline Cardiovascular Diabetology & 7.332 & Open Access & 3,170 & Case by case \\
\hline Cardiovascular Diagnosis and Therapy & 2.615 & Open Access & 1,390 & Yes, upon request \\
\hline Cardiovascular Digital Health Journal & - & Open Access & 2,800 & Automatic (Research4Life) \\
\hline Cardiovascular Drugs and Therapy & 4.069 & Hybrid & 3,860 & No \\
\hline Cardiovascular Engineering and Technology & 1.771 & Hybrid & 3,260 & No \\
\hline Cardiovascular Journal of Africa & 0.897 & Open Access & 461 & No \\
\hline Cardiovascular Pathology & 1.756 & Hybrid & 3,000 & Case by case (Research4Life) \\
\hline Cardiovascular Research & 8.168 & Hybrid & 4,592 & No \\
\hline Cardiovascular Therapeutics & 2.538 & Open Access & 2,100 & Automatic \\
\hline Cardiovascular Toxicology & 2.284 & Hybrid & 3,860 & No \\
\hline Cardiovascular Ultrasound & 2.051 & Open Access & 2,790 & Case by case \\
\hline $\begin{array}{l}\text { Catheterization and Cardiovascular } \\
\text { Interventions }\end{array}$ & 2.044 & Hybrid & 3,800 & No \\
\hline
\end{tabular}




\begin{tabular}{|c|c|c|c|c|}
\hline Circulation & 23.603 & Hybrid & 3,450 & No \\
\hline $\begin{array}{l}\text { Circulation: Arrhythmia and } \\
\text { Electrophysiology }\end{array}$ & 4.393 & Hybrid & 3,450 & No \\
\hline Circulation: Cardiovascular Imaging & 5.691 & Hybrid & 3,450 & No \\
\hline Circulation: Cardiovascular Interventions & 5.493 & Hybrid & 3,450 & No \\
\hline $\begin{array}{l}\text { Circulation: Cardiovascular Quality \& } \\
\text { Outcomes }\end{array}$ & 5.071 & Hybrid & 3,450 & No \\
\hline $\begin{array}{l}\text { Circulation: Genomic and Precision } \\
\text { Medicine }\end{array}$ & 4.534 & Hybrid & 3,450 & No \\
\hline Circulation: Heart Failure & 6.033 & Hybrid & 3,450 & No \\
\hline Circulation Research & 14.467 & Hybrid & 3,450 & No \\
\hline Circulation Journal & 2.540 & Open Access & 1,929 & No \\
\hline Clinical Cardiology & 2.248 & Open Access & 2,475 & Yes, upon request \\
\hline Clinical Research in Cardiology & 5.268 & Hybrid & 3,760 & No \\
\hline $\begin{array}{l}\text { Clinical Research in Cardiology } \\
\text { Supplements }\end{array}$ & - & Hybrid & 2,760 & No \\
\hline Congenital Heart Disease & 1.663 & Open Access & 1,200 & Case by case \\
\hline Coronary Artery Disease & 1.335 & Hybrid & 2,400 & No \\
\hline Current Cardiology Reports & 2.434 & Hybrid & 3,860 & No \\
\hline Current Opinion in Cardiology & 2.149 & Hybrid & 3,000 & No \\
\hline Current Problems in Cardiology & 2.966 & Hybrid & 3,000 & Case by case (Research4Life) \\
\hline e-Journal of Cardiology Practice & - & Open Access & $\begin{array}{c}\text { Free (invited } \\
\text { submissions only) }\end{array}$ & - \\
\hline Echocardiography & 1.393 & Hybrid & 2,700 & No \\
\hline ESC Heart Failure & 3.902 & Open Access & 3,366 & Case by case (Research4Life) \\
\hline Eurolntervention & 3.993 & Open Access & Free & - \\
\hline EP Europace & 4.045 & Hybrid & 4,592 & No \\
\hline European Heart Journal & 22.673 & Hybrid & 4,592 & No \\
\hline $\begin{array}{l}\text { European Heart Journal: Acute } \\
\text { Cardiovascular Care }\end{array}$ & 3.813 & Hybrid & 3,300 & No \\
\hline $\begin{array}{l}\text { European Heart Journal: Cardiovascular } \\
\text { Imaging }\end{array}$ & 4.841 & Hybrid & 4,549 & No \\
\hline $\begin{array}{l}\text { European Heart Journal: Cardiovascular } \\
\text { Pharmacotherapy }\end{array}$ & 6.696 & Hybrid & 3,830 & No \\
\hline European Heart Journal: Case Reports & - & Open Access & 726 & Yes, upon request \\
\hline European Heart Journal: Digital Health & - & Open Access & 3,225 & Yes, upon request \\
\hline $\begin{array}{l}\text { European Heart Journal: Quality of Care } \\
\text { and Clinical Outcomes }\end{array}$ & - & Hybrid & 4,280 & No \\
\hline European Heart Journal Supplements & 1.655 & Hybrid & 4,592 & No \\
\hline European Journal of Cardiovascular Nursing & 2.296 & Hybrid & 3,300 & No \\
\hline European Journal of Heart Failure & 11.627 & Hybrid & 4,300 & No \\
\hline European Journal of Preventive Cardiology & 5.864 & Hybrid & 3,300 & No \\
\hline Frontiers in Cardiovascular Medicine & 3.915 & Open Access & 2,490 & Case by case \\
\hline
\end{tabular}




\begin{tabular}{|c|c|c|c|c|}
\hline Global Heart & 3.862 & Open Access & 1,583 & Automatic discount \\
\hline Heart & 5.213 & Hybrid & 3,100 & No \\
\hline Heart and Vessels & 1.618 & Hybrid & 3,260 & No \\
\hline Heart Failure Clinics & 2.327 & Hybrid & 2,680 & Case by case (Research4Life) \\
\hline Heart Failure Reviews & 3.538 & Hybrid & 3,860 & No \\
\hline Heart \& Lung & 1.630 & Hybrid & 2,860 & Case by case (Research4Life) \\
\hline Heart, Lung and Circulation & 2.194 & Hybrid & 2,500 & Case by case (Research4Life) \\
\hline Heart Rhythm & 5.731 & Hybrid & 3,500 & Case by case (Research4Life) \\
\hline Heart Rhythm: Case Reports & - & Open Access & 1,050 & Automatic (Research4Life) \\
\hline Heart Rhythm $\mathrm{O}^{2}$ & - & Open Access & 2,150 & Automatic (Research4Life) \\
\hline Hellenic Journal of Cardiology & 4.047 & Open Access & Free & - \\
\hline Herz & 1.033 & Hybrid & 2,760 & No \\
\hline International Heart Journal & 1.906 & Open Access & 1,447 & No \\
\hline International Journal of Cardiology & 3.229 & Hybrid & 3,470 & Case by case (Research4Life) \\
\hline $\begin{array}{l}\text { International Journal of Cardiology: } \\
\text { Congenital Heart Disease }\end{array}$ & - & Open Access & 2,000 & Automatic (Research4Life) \\
\hline $\begin{array}{l}\text { International Journal of Cardiology: Heart } \\
\text { and Vasculature }\end{array}$ & - & Open Access & 2,550 & Automatic (Research4Life) \\
\hline $\begin{array}{l}\text { International Journal of Cardiovascular } \\
\text { Imaging }\end{array}$ & 1.969 & Hybrid & 3,260 & No \\
\hline JAMA Cardiology & 12.794 & Hybrid & 5,000 & No \\
\hline Journal of Cardiology & 2.246 & Hybrid & 3,000 & Case by case (Research4Life) \\
\hline $\begin{array}{l}\text { Journal of Cardiopulmonary Rehabilitation } \\
\text { and Prevention }\end{array}$ & 1.383 & Hybrid & 3,375 & No \\
\hline $\begin{array}{l}\text { Journal of Cardiovascular Computed } \\
\text { Tomography }\end{array}$ & 2.892 & Hybrid & 3,150 & Case by case (Research4Life) \\
\hline Journal of Cardiovascular Electrophysiology & 2.424 & Hybrid & 3,900 & No \\
\hline $\begin{array}{l}\text { Journal of Cardiovascular Magnetic } \\
\text { Resonance }\end{array}$ & 5.361 & Open Access & 2,680 & Case by case \\
\hline Journal of Cardiovascular Medicine & 1.225 & Hybrid & 2,800 & No \\
\hline Journal of Cardiovascular Nursing & 1.675 & Hybrid & 2,570 & No \\
\hline Journal of Cardiovascular Pharmacology & 2.598 & Hybrid & 3,150 & No \\
\hline $\begin{array}{l}\text { Journal of Cardiovascular Pharmacology } \\
\text { and Therapeutics }\end{array}$ & 2.322 & Hybrid & 3,000 & No \\
\hline $\begin{array}{l}\text { Journal of Cardiovascular Translational } \\
\text { Research }\end{array}$ & 3.312 & Hybrid & 3,860 & No \\
\hline Journal of Electrocardiology & 0.944 & Hybrid & 2,830 & Case by case (Research4Life) \\
\hline Journal of Geriatric Cardiology & 2.491 & Open Access & Free & - \\
\hline $\begin{array}{l}\text { Journal of Interventional Cardiac } \\
\text { Eletrophysiology }\end{array}$ & 1.277 & Hybrid & 3,260 & No \\
\hline Journal of Interventional Cardiology & 1.758 & Open Access & 2,100 & Automatic \\
\hline Journal of Invasive Cardiology & 1.470 & Open Access & Free & - \\
\hline $\begin{array}{l}\text { Journal of Molecular and Cellular } \\
\text { Cardiology }\end{array}$ & 4.133 & Hybrid & 3,320 & Case by case (Research4Life) \\
\hline
\end{tabular}




\begin{tabular}{|c|c|c|c|c|}
\hline Journal of Nuclear Cardiology & 3.366 & Hybrid & 3,860 & No \\
\hline Journal of the American Heart Association & 4.605 & Open Access & 2,750 & Automatic \\
\hline $\begin{array}{l}\text { Journal of the American College of } \\
\text { Cardiology (JACC) }\end{array}$ & 20.589 & Hybrid & 3,000 & Case by case (Research4Life) \\
\hline JACC: Basic to Translational Science & - & Open Access & 3,400 & Automatic (Research4Life) \\
\hline JACC: CardioOncology & - & Open Access & 2,500 & Automatic (Research4Life) \\
\hline JACC: Cardiovascular Imaging & 12.740 & Hybrid & 3,000 & Case by case (Research4Life) \\
\hline JACC: Cardiovascular Interventions & 8.432 & Hybrid & 3,000 & Case by case (Research4Life) \\
\hline JACC: Case Reports & - & Open Access & 600 & Automatic (Research4Life) \\
\hline JACC: Clinical Electrophysiology & - & Hybrid & 3,000 & Case by case (Research4Life) \\
\hline JACC Heart Failure & 9.140 & Hybrid & 2,500 & Case by case (Research4Life) \\
\hline Journal of Cardiac Failure & 3.623 & Hybrid & 3,040 & Case by case (Research4Life) \\
\hline $\begin{array}{l}\text { Journal of the American Society of } \\
\text { Echocardiography }\end{array}$ & 5.508 & Hybrid & 3,000 & Case by case (Research4Life) \\
\hline Journal of Thrombosis and Thrombolysis & 2.054 & Hybrid & 3,860 & No \\
\hline Kardiologia Polska & 1.874 & Open Access & Free & - \\
\hline Kardiologiya & 0.264 & Subscription & - & - \\
\hline Korean Circulation Journal & 2.322 & Open Access & Free & - \\
\hline Minerva Cardioangiologica & 0.713 & Subscription & - & - \\
\hline Nature Reviews Cardiology & 20.260 & $\begin{array}{l}\text { Hybrid (as of } \\
\text { 2021) }\end{array}$ & Not yet available & No \\
\hline Netherlands Heart Journal & 1.933 & Open Access & Free & - \\
\hline $\begin{array}{l}\text { Nutrition Metabolism and Cardiovascular } \\
\text { Diseases }\end{array}$ & 3.700 & Hybrid & 3,000 & Case by case (Research4Life) \\
\hline Open Heart & - & Open Access & 2,628 & Case by case \\
\hline Pace-Pacing and Clinical Electrophysiology & 1.303 & Hybrid & 3,200 & No \\
\hline Pediatric Cardiology & 1.564 & Hybrid & 3,260 & No \\
\hline Perfusion-United Kingdom & 1.234 & Hybrid & 3,000 & No \\
\hline Postepy w Kardiologii Interwencyjnej & 1.347 & Open Access & 244 & No \\
\hline Progress in Cardiovascular Diseases & 6.763 & Hybrid & 2,800 & Case by case (Research4Life) \\
\hline Pulmonary Circulation & 2.205 & Open Access & 2,000 & Case by case \\
\hline Respiratory Medicine & 3.095 & Hybrid & 3,500 & Case by case (Research4Life) \\
\hline Reviews in Cardiovascular Medicine & 0.659 & Open Access & 1,250 & Case by case \\
\hline Revista Española de Cardiología (REC) & 4.642 & Hybrid & 3,000 & Case by case (Research4Life) \\
\hline REC: CardioClinics & - & Hybrid & 1,500 & Case by case (Research4Life) \\
\hline REC: Interventional Cardiology & - & Open Access & Free & - \\
\hline Revista Portuguesa de Cardiologia & 0.960 & Open Access & Free & - \\
\hline Scandinavian Cardiovascular Journal & 1.084 & Hybrid & 2,995 & No \\
\hline Texas Heart Institute Journal & 1.023 & Open Access & Free & - \\
\hline Trends in Cardiovascular Medicine & 4.755 & Hybrid & 3,300 & Case by case (Research4Life) \\
\hline
\end{tabular}

$\mathrm{BMC}=$ BioMed Central; EP Europace=The European Journal of Pacing, Arrhythmias and Cardiac Electrophysiology; ESC=European Society of Cardiology; JAMA=Journal of the American Medical Association; USD=U.S. Dollar 
Supplemental Table 2. Publishing models and article processing charges for cardiac surgery journals indexed in InCites Journal Citation Reports.

\begin{tabular}{|c|c|c|c|c|}
\hline Journal Name & $\begin{array}{l}\text { Impact } \\
\text { Factor }\end{array}$ & $\begin{array}{l}\text { Publishing } \\
\text { Model }\end{array}$ & $\begin{array}{l}\text { Article Processing } \\
\text { Charges (USD) }\end{array}$ & Waivers Available \\
\hline Annals of Cardiothoracic Surgery & 3.058 & Open access & 2,390 & No \\
\hline $\begin{array}{l}\text { Annals of Thoracic and Cardiovascular } \\
\text { Surgery }\end{array}$ & 1.584 & Open access & 383 & No \\
\hline Annals of Thoracic Surgery & 3.639 & Hybrid & 3,000 & Case by case (Research4Life) \\
\hline Brazilian Journal of Cardiovascular Surgery & 1.053 & Open access & Free & - \\
\hline $\begin{array}{l}\text { European Journal for Cardio-Thoracic } \\
\text { Surgery }\end{array}$ & 3.486 & Hybrid & 3,087 & No \\
\hline $\begin{array}{l}\text { General Thoracic and Cardiovascular } \\
\text { Surgery }\end{array}$ & 1.088 & Hybrid & 3,260 & No \\
\hline Heart Surgery Forum & 0.404 & Open Access & 950 & No \\
\hline $\begin{array}{l}\text { Interactive CardioVascular and Thoracic } \\
\text { Surgery }\end{array}$ & 1.675 & Open Access & Free & - \\
\hline Journal of Cardiac Surgery & 1.490 & Hybrid & 2,900 & No \\
\hline $\begin{array}{l}\text { Journal of Cardiothoracic and Vascular } \\
\text { Anesthesia }\end{array}$ & 2.258 & Hybrid & 2,680 & Case by case (Research4Life) \\
\hline Journal of Cardiothoracic Surgery & 1.506 & Open Access & 2,490 & Case by case \\
\hline Journal of Cardiovascular Surgery & 1.415 & Subscription & - & - \\
\hline Journal of Heart and Lung Transplantation & 7.865 & Hybrid & 3,300 & Case by case (Research4Life) \\
\hline $\begin{array}{l}\text { Journal of Thoracic and Cardiovascular } \\
\text { Surgery (JTCVS) }\end{array}$ & 4.451 & Hybrid & 2,950 & Case by case (Research4Life) \\
\hline JTCVS Open & - & Open Access & 2,000 & Automatic (Research4Life) \\
\hline JTCVS Techniques & - & Open Access & 2,000 & Automatic (Research4Life) \\
\hline $\begin{array}{l}\text { Multimedia Manual of Cardio-Thoracic } \\
\text { Surgery }\end{array}$ & - & Open Access & Free & - \\
\hline $\begin{array}{l}\text { Operative Techniques in Thoracic and } \\
\text { Cardiovascular Surgery }\end{array}$ & - & Hybrid & 2,740 & Case by case (Research4Life) \\
\hline $\begin{array}{l}\text { Seminars in Thoracic and Cardiovascular } \\
\text { Surgery }\end{array}$ & 2.133 & Hybrid & 2,740 & Case by case (Research4Life) \\
\hline $\begin{array}{l}\text { Seminars in Thoracic and Cardiovascular } \\
\text { Surgery: Pediatric Cardiac Surgery Annual }\end{array}$ & - & Hybrid & 3,170 & Case by case (Research4Life) \\
\hline Thoracic and Cardiovascular Surgeon & 1.209 & Subscription & - & - \\
\hline $\begin{array}{l}\text { Thoracic and Cardiovascular Surgeon } \\
\text { Reports }\end{array}$ & - & Open Access & 2,300 & No \\
\hline
\end{tabular}

USD=U.S. Dollar

defined on the basis of authors' affiliation, which may limit authors from low- and middle-income countries who have recently moved to or are studying or training in high-income countries, authors from lower socioeconomic status, or authors without research funding (e.g., non-doctoral graduate students, residents) whose institution is not based in eligible countries. Some journals offer minimal discounts (e.g., 10-15\% discount) to partnering institutions, which may perpetuate barriers for those without funding and only skew the dissemination of research towards those with research funding. While some smaller journals are independently operating or associated with non-profit organizations or professional societies, most journal have contracts with large publishing entities that jointly comprise the billion-dollar academic publishing industry, wherein profit margins run up to $30 \% \%^{[2]}$. Few OA or hybrid journals had no APCs and were most commonly covering or based in Latin America, where free-of-charge OA journals are commonplace and supported by both professional society and governmental support, a model that may be extended elsewhere ${ }^{[10]}$. 
Publishing does not come without a cost, as journals and publishers must hire editorial staff, manage online platforms and servers, maintain publication relations, and ensure typesetting of accepted publications. Nevertheless, they equally rely on the voluntary time of reviewers and most editors, whereas societal, industry, and other partnerships typically result in substantial monetary and in-kind support for journals to cover large shares of the fixed costs. The true costs of publishing a single article remain unclear, but estimates have shown to be as low as US\$300 per article, far lower than the requested APCS ${ }^{[11]}$.

The commonly cited publish-or-perish mentality in academic medicine has led to an increase in the number of predatory journals, seeking to take advantage of vulnerable authors facing barriers to publishing in acknowledged journals or wanting to find a quick way to publish. Previously known cardiovascular journals have been bought to leverage a pre-existing reputation and attract clinicians and researchers, yet they only publish verbatim (i.e., not reviewing or editing submissions), generating tens up to hundreds of thousands of dollars per journal per year ${ }^{[12]}$. What is especially concerning is the potentially important knowledge remaining undetected and not disseminated as a result of such predatory journals. For example, the field of global cardiac surgery is nascent, and local studies reporting on cardiac surgery outcomes in lowand middle-income countries have been identified in predatory journals. Barriers for authors from these countries are upheld by high APCs (less able to publish in certain journals) and non-OA (less able to access articles), underscoring the need to change the academic publishing narrative.

\section{Limitations}

Several limitations are inherent to this analysis. We used JCR to identify established journals and their sister journals, but we are aware of a number of journals not listed on JCR that are legitimate (e.g., PubMed/MEDLINE indexing, societal affiliation). However, as the number of journals included on JCR is large, our analysis paints a representative picture. Moreover, various journals give discounts for society members or on a case-by-case basis, which may lead to a vast variety in the exact APCs to be paid for some authors. Lastly, we utilized the highest APCs per journal, which sometimes have lower APCs for article types other than original articles, such as case reports. However, as most original research is published as original articles, we believe this most accurately reflects the status of authors.

\section{CONCLUSION}

OA publishing is common across cardiology and cardiac surgery journals with substantial APCs. Fees are prohibitive for unfunded and lesser-funded researchers in the absence of broader waiver considerations.

\section{No financial support. \\ No conflict of interest.}

\section{Authors' roles \& responsibilities}

DV Substantial contributions to the conception or design of the work; or the acquisition, analysis, or interpretation of data for the work; drafting the work or revising it critically for important intellectual content; final approval of the version to be published

JGYL Drafting the work or revising it critically for important intellectual content; final approval of the version to be published

MPBOS Drafting the work or revising it critically for important intellectual content; final approval of the version to be published

EWE Substantial contributions to the conception or design of the work; or the acquisition, analysis, or interpretation of data for the work; drafting the work or revising it critically for important intellectual content; final approval of the version to be published

\section{REFERENCES}

1. Till BM, Rudolfson N, Saluja S, Gnanaraj J, Samad L, Ljungman D, et al. Who is pirating medical literature? A bibliometric review of 28 million Sci-Hub downloads. Lancet Glob Health. 2019;7(1):e30-1. doi:10.1016/ S2214-109X(18)30388-7.

2. Nabyonga-Orem J, Asamani JA, Nyirenda T, Abimbola S. Article processing charges are stalling the progress of African researchers: a call for urgent reforms. BMJ Glob Health. 2020;5(9):e003650. doi:10.1136/ bmjgh-2020-003650.

3. Grudniewicz A, Moher D, Cobey KD, Bryson GL, Cukier S, Allen K, et al. Predatory journals: no definition, no defence. Nature. 2019;576(7786):2102. doi:10.1038/d41586-019-03759-y.

4. Vervoort D, Ma X, Shrime MG. Money down the drain: predatory publishing in the COVID-19 era. Can J Public Health. 2020;111(5):6656. doi:10.17269/s41997-020-00411-5.

5. Björk BC. Growth of hybrid open access, 2009-2016. PeerJ. 2017;5:e3878. doi:10.7717/peerj.3878.

6. Nyamai LA, Jin YP, Mathew DJ, Dharia RS, Trope GE, Buys YM. The cost of publishing in an indexed ophthalmology journal in 2019. Can J Ophthalmol. 2020;55(6):518-23. doi:10.1016/j.jcjo.2020.06.023.

7. Bakker C, Stephenson C, Stephenson E, Chaves D. Public funding and open access to research: a review of Canadian multiple sclerosis research. J Med Internet Res. 2017;19(2):e52. doi:10.2196/jmir.6250.

8. Smith E, Haustein S, Mongeon P, Shu F, Ridde V, Larivière V. Knowledge sharing in global health research - the impact, uptake and cost of open access to scholarly literature. Health Res Policy Syst. 2017;15(1):73. doi:10.1186/s12961-017-0235-3.

9. Solomon D, Björk BC. Article processing charges for open access publication-the situation for research intensive universities in the USA and Canada. PeerJ. 2016;4:e2264. doi:10.7717/peerj.2264.

10. Debat H, Babini D. Plan S: take Latin America's long experience on board. Nature. 2019;573(7775):495. doi:10.1038/d41586-019-02857-1.

11. Van Noorden R. Open access: the true cost of science publishing. Nature. 2013;495(7442):426-9. doi:10.1038/495426a. Erratum in: Nature. 2013;496(7444):151. Erratum in: Nature. 2013;499(7456):19.

12. Spears T. Canadian cardiac journal turns "predatory". CMAJ. 2014;186(14):E525. doi:10.1503/cmaj.109-4889. 